\title{
FUZZYSS'2011: 2nd International Fuzzy Systems Symposium 17-18 November 2011, Ankara, Turkey
}

\author{
Adil Baykasoglu ${ }^{\mathrm{a}, *}$, Candan Gokceoglu ${ }^{\mathrm{b}}$, Turkay Dereli ${ }^{\mathrm{c}}$ and I. Burhan Turksen ${ }^{\mathrm{d}}$ \\ ${ }^{a}$ Dokuz Eylul University, Izmir, Turkey \\ ${ }^{\mathrm{b}}$ Hacettepe University, Hacettepe, Ankara, Turkey \\ ${ }^{\mathrm{c}}$ University of Gaziantep, Gaziantep, Turkey \\ ${ }^{\mathrm{d}}$ TOBB Economy and Technology University, Ankara, Turkey
}

Welcome to the Journal of Intelligent and Fuzzy Systems's special issue for "FUZZYSS'2011: 2nd International Fuzzy Systems Symposium".

Since its introduction by Prof. Dr. Lotfi A. Zadeh fuzzy logic, fuzzy sets and systems have been applied to various problems in many diverse areas. As everything try to evolve towards better states, the fuzzy theory and its applications are also evolving to model and solve more complicated problems. However, as noted by many experts, it may not be straightforward to differentiate between problems that really require a crisp or fuzzy modeling and solution approach. In fact, the boundary between them may also be uncertain. What sort of problems really requires fuzzy modeling and solution? What is "fuzziness" in them? Without appropriately answering these basic questions, application of fuzzy logic may not be very helpful. As noted by Prof. Zadeh; "Fuzziness describes the ambiguity of an event, whereas randomness describes the uncertainty in the occurrence of the event". Therefore, it is very important to clearly explore the type of uncertainly in any application before proving a model and/or solution to it.

\footnotetext{
${ }^{*}$ Corresponding author. Adil Baykasoglu, Faculty of Engineering, Department of Industrial Engineering, Dokuz Eylul University, Tinaztepe Campus, 35160 Buca, Izmir, Turkey. Tel.: +90 23 2412-7600; Fax: +90 23 2412-7608. E-mails: baykasoglu @gmail.com; adil.baykasoglu@deu.edu.tr.
}

We should say that scientific research related to fuzzy logic and its extensions is still an open and very promising active research area. There is still much to explore. In this special issue the readers will find some of the innovative applications of fuzzy sets and systems to several interesting problems from very diverse areas.

The special issue provides some of the good quality original research papers, which were presented orally and discussed at the "FUZZYSS'2011: 2nd International Fuzzy Systems Symposium, 17-18 November 2011 Ankara, Turkey", were selected as candidates for this special issue. After the re-submission of the revised/extended manuscripts, and rigorous refereeing processes, seven papers were finally considered for the publication in the scheduled special issue of Journal of Intelligent and Fuzzy Systems. We would like to thank to Prof. Reza Langari (the editor of Journal of Intelligent and Fuzzy Systems) for giving us the opportunity to prepare this special issue. This special issue provides the reader a collection of seven articles, which offers an exclusive perspective on the topics ranging from fuzzy clustering, fuzzy assembly line balancing, developing sampling strategies with neuro-fuzzy systems, daily streamflow prediction with fuzzy logic, structural verification through fuzzy sets for clinical guidelines, prediction of technology commercialization with fuzzy approaches, and fuzzy medium-term planning in closed-loop supply chains. 